\title{
Observations sur la zonation des cours d'eau de la savane ouest- africaine : Trichoptères du Sud-Ouest du Burkina Faso
}

\author{
F.-M. Gibon 1 \\ W. Guenda 2 \\ B. Coulibaly ${ }^{3}$
}

Mots-clés : Afrique occidentale, Trichoptera, Hydropsychidae, Ecnomidae, Hydroptilidae, taxinomie, biogéographie, zonation.

Plusieurs études réalisées dans l'ouest du Burkina, sur les hauts cours du Kou, du Mouhoun (bassin des Voltas) et de la Léraba (bassin de la Comoé), ont permis la mise en évidence d'une région faunistique originale. Ces travaux ont notablement accru notre connaissance de la faune des Trichoptères de ce pays. Les recherches antérieures menées soit au Burkina sur le Nazinon et la Comoé, soit dans des écosystèmes similaires en Côte d'Ivoire, indiquaient une remarquable homogénéité faunistique des cours d'eau situés dans la zone des savanes. Par contre, nous observons, dans cette zone refuge, la présence d'espèces dont les affinités sont forestières ou montagnardes (Fouta Djalon). Ce phénomène est lié à une altitude supérieure, à la permanence de l'écoulement et à la présence de forêts classés. Huit espèces nouvelles sont décrites, elles appartiennent aux genres Cheumatopsyche, Ecnomus, Hydroptila, Oxyethira et Ugandatrichia.

Observations on the zonation of running waters of the west-African savannas : Trichoptera from the Southwest of Burkina Faso

Keywords : West-Africa, Trichoptera, Hydropsychidae, Ecnomidae, Hydroptilidae, taxonomy, biogeography, zonation.

A new faunistic area is reported from the upper courses of the Kou, the Mouhoun (tributaries of the Volta) and the Leraba (tributary of the Comoe). Our knowledge of the caddis fauna of the Western Burkina is synthetized ; eight new species are described, they belong to the genera Cheumatopsyche, Ecnomus, Hydroptila, Oxyethira and Ugandatrichia. Though previous studies have shown a great homogeneity of the savannas, we observe here species only known from forest or mountain areas. Probable causes are : a greater altitude, the permanence of flow and the presence of protected forests.

\section{Le problème et son intérêt}

La connaissance des Trichoptères du Burkina Faso s'est développée dans le cadre des recherches suscitées par le Programme de lutte contre l'Onchocercoce (O.C.P.). L'objectif principal était l'étude des effets des traitements insecticides antisimulidiens sur les biocénoses lotiques (Guenda 1985, Yamego et al. 1988). A quelques exceptions près (notamment les Simuliidae, Elouard 1983), le faible niveau des connaissances taxonomiques disponibles lors du

1. Orstom, Département Eaux Continentales, 213, rue La Fayette, 75010 Paris, France.

2. Institut des Sciences de l'Education, Université de Ouagadougou, B.P. 7021, Burkina Faso.

3. Unité d'Hydrobiologie, Programme de lutte contre l'Onchocercose, O.M.S./O.C.P., B.P. 549 Ouagadougou, Burkina Faso. début des opérations de lutte (milieu des années soixante-dix) ne permettait pas de travailler au niveau spécifique. Or certaines questions nécessitent ce niveau. Deux d'entre elles présentaient un intérêt immédiat. La première est la généralisation géographique des résultats obtenus sur les sites de recherche et de surveillance écologique, question qui concerne la plupart des études d'impact. La seconde est l'hypothèse de la recolonisation des zones traitées par la faune des petits affluents ; cette question est particulière à ce programme. En effet, les vecteurs (Simulium damnosum s. l. Théobald, 1903) ne colonisent pas les cours supérieurs, ni les plus petits affluents. Ceux-ci ne sont donc pas soumis aux épandages de larvicides (Le Berre 1966); la limite est variable d'une année à l'autre, en fonction des conditions hydrologiques. Dans l'éventualité d'un 
impact faunistique drastique des campagnes de lutte, ces milieux constitueraient un ensemble de refuges à partir desquels se produirait un retour à la situátion originelle. Cette hypothèse suppose un fort recouvrement de la faune des rivières et de celle du réseau des petits affluents qui, en général, n'existe pas. La plupart des études de zonation mettent en évidence, a contrario, des faunes rhitriques et potamiques plus ou moins distinctes (Botosaneanu 1979, Wasson 1989, Zwick 1992). Cependant, un tel phénomène a été observé dans les bassins du Bandama (Gibon \& Statzner, 1985) et de la Volta (Guenda, sous presse). Ces études indiquent que, dans les savanes soudaniennes, règne généralement une forte homogénéité faunistique. La distribution des insectes lotiques est déterminée par un ensemble de conditions liées au biome et non au « stream order » Il s'agit d'une situation atypique dont l'étude mériterait d'être approfondie.

Parmi les raisons avancées pour expliquer cette situation figurent l'intermittence de l'écoulement et le faible gradient d'altitude rencontré dans le bassin du Bandama. Il était donc intéressant de pouvoir étudier une faune lotique dans ces savanes (et de ce fait, dans les mêmes conditions climatiques) mais à des altitudes plus élevées. C'est le cas des cours supérieurs du Kou et du Mouhoun (bassin des Voltas), ainsi que ceux de la Comoé et de la Léraba (bassin de la Comoé). Ils sont situés sur le plateau qui s'étend à l'ouest de Bobo-Dioulasso et Banfora, à une altitude qui varie de 350 à $550 \mathrm{~m}$ (les sommets atteignent 600 à $750 \mathrm{~m}$ ), soit, en moyenne, une centaine de mètres plus haut que les cours supérieurs du Nzi/Bandama. Nous y avons effectivement observé une faune différente et parfois inattendue. L'ensemble de ces observations, confronté aux donnée disponibles sur les régions voisines, ainsi qu'aux connaissances sur les aires de distribution des espèces, met en évidence une frontière faunistique. Celleci se traduit par un schéma de zonation longitudinale plus proche du modèle classique.

\section{Matériel et méthodes}

Nous avons réalisé une première synthèse des connaissances faunistiques, écologiques et biogréographiques disponibles, de façon à orienter les travaux futurs. L'impératif spécifique, essentiel dans l'optique de ces recherches, a considérablement restreint notre champ d'étude. Nous l'avons limité aux
Hydropsychidae, Philopotamidae, Ecnomidae et Hydroptilidae, familles pour lesquelles nous disposons, non seulement de l'identification spécifique mais aussi d'une première approche des distributions en Afrique occidentale. Les genres Orthotrichia et Psychomyiellodes ont été exclus car nous n'avons pas encore une connaissance suffisante de ces groupes au niveau régional. Nous avons travaillé sur des adultes capturés principalement au piège lumineux (U.V. ou lumière noire). Ce choix s'explique, d'une part par l'impossibilité d'identifier actuellement les larves au niveau spécifique, d'autre part par la présence continue des imagos au bord des cours d'eau permanents, ce qui rend leur échantillonnage beaucoup plus commode que dans les milieux tempérés. Les cartes de distribution sont établies à partir de captures qui ont été réalisées par l'Orstom ou dans le cadre d'O.C.P. La plupart de ces données sont originales, car les rares éléments bibliographiques antérieurs sont parfois difficiles à localiser précisément.

\section{Bilan des connaissances faunistiques sur les Trichoptères du Burkina Faso (Tableau 1)}

Les premières données concernant les groupes étudiés ici ont été publiées par Marlier (1978) puis Guenda (1985 et 1986/87). Elles concernent le Nazinon (ex Volta Rouge) dans le secteur du pont du Pô et plus en amont vers Sakoinse. Une mission effectuée en Novembre 1989 (F.-M. G. et B. C.) nous a fourni également quelques éléments sur la faune du Nazinon et nous a permis d'étudier la Bougouriba (affluent du Mouhoun) dans la région de Diébougou. Enfin des données (assez nombreuses et non publiées) concernent la Léraba, la Comoé et leurs affluents, dans un secteur qui s'étend du pont frontière (sur la route Bobo Dioulasso / Ferkéssédougou) jusqu'aux rapides dits "Gréchan » (au nord du Parc National de la Comoé). Ces zones sont localisées sur la figure 46. L'ensemble des résultats est présenté dans le tableau 1.

Il apparait que ces trois secteurs ont des peuplements semblables. Ce résultat est plus net si on le compare à la faune du nord de la Côte d'Ivoire (en particulier le bassin du Bandama) qui est relativement bien connue. Statzner (1984) définit chez les Cheumatopsyche, un groupe caractéristique des savanes, composé de $C$. falcifera (Ulmer), 1930, $C$. digitata (Mosely), 1935, et C. copiosa Kimmins, 
Tableau 1. Trichoptères du Burkina Faso (part.), données antérieures.

Table 1. Trichoptera of Burkina Faso (part.), previous data.

\begin{tabular}{|l|c|c|c|}
\hline & 1 & 2 & 3 \\
\hline Cheumatopsyche lesnei & & + & + \\
\hline Cheumatopsyche copiosa & + & + & + \\
\hline Cheumatopsyche falcifera & + & & + \\
\hline Cheumatopsyche digitata & + & + & + \\
\hline Aethaloptera dispar s.l. & + & + & + \\
\hline Polymorphanisus angust. & + & & \\
\hline Amphipsyche senegalensis & + & + & + \\
\hline Chimarra minima & + & & + \\
\hline Chimarra sassandrae & + & + & \\
\hline Ecnomus ulmeri & + & & + \\
\hline Ecnomus dinderessoi & + & & \\
\hline Ecnomus wendmanegrei & + & & \\
\hline Ecnomus foliatus curvatus & & + & \\
\hline Hydroptila hirra & + & & \\
\hline Catoxyethira mali & + & & \\
\hline Oxyethira sp. & & + & + \\
\hline $1 \quad$ Léraba / Comoé & & & \\
\hline $2 \quad$ Bougouriba & & & \\
\hline $3 \quad$ Nazinon & & & \\
\hline
\end{tabular}

1956. C. lesnei (Mosely), 1932, n'était alors connu que par quelques larves qui ont été identifiées par la suite (Guenda 1985). La figure 47 indique les sites de captures de cette dernière espèce ; ils se situent strictement dans les zones sahéliennes et soudaniennes. Chez les Chimarra, Gibon (1985) met en évidence un groupe caractéristique des savanes, composé de C. minima Ulmer, 1907, et de C. sassandrae Gibon, 1982. Chez les Macronematinae, la faune burkinabe est un peu moins riche que ce que l'on pouvait prévoir à partir des résultats de Statzner \& Gibon (1984), en particulier dans les genres Macrostemum et Protomacronema. Cependant, les espèces présentes sont, ici encore, celles qui dominent sur le Nord du Bandama; en particulier, Amphipsyche senegalensis (Brauer), 1875, et Aethaloptera dispar Brauer, 1875. Nos connaissances sur les Ecnomidae et les Hydroptilidae sont plus récentes et probablement encore incomplètes. La présence des genres Hydroptila, Oxyethira et Catoxyethra est constatée sur le Nazinon mais les espèces n'ont pu être identifiées. Sur la Léraba, on observe $H$. hirra Mosely, 1948, une espèce ubiquiste très largement répandue (jusque dans l'Aïr, un massif saharien), ainsi que $C$. mali (Marlier), 1978, l'une des deux espèces de Catoxyethira (avec $C$. veruta septentrionalis Gibon, 1985) susceptibles de coloniser les cours d'eau des savanes (Gibon, 1985). Ecnomus ulmeri Mosely, 1932, est l'espèce la plus répandue en Afrique occidentale $; E$. dinderessoi $n$. sp. est connu sur les bassins du Niger (Milo, Dion), du Bandama, du Sénégal (Bakoye, Falémé) et de la Kolenté ; $E$. wendmanegrei $n$. sp. sur les bassins du Bandama, du Sassandra, du Niger (Milo), de la Kolenté et du Ouémé.

En conclusion, les trois régions qui étaient inventoriées au Burkina présentent une faune caractéristique des savanes, constituée d'espèces à vaste aire de répartition. Elles indiquaient une remarquable continuité faunistique entre le nord du bassin du Bandama, celui de la Comoé et celui de la Volta. Aucune frontière faunistique (d'origine écologique ou géographique) n'était, à ce stade, mise en évidence.

\section{L'originalité faunistique du plateau de Bobo-Dioulasso / Banfora}

Des prospections ont été réalisées en Novembre 1989 (sites b et $c$ de la figure 45, F.-M. G. et B. C.), en Février et Décembre 1991 (sites e, f, g et $i$, W. G.) ainsi qu'en Janvier 1993 (sites a, d et h, F.-M. G. et B. C.), c'est-à-dire entre la fin de la saison des pluies et la période de sécheresse maximale. La figure 45 localise les sites de prospections où ont été capturées des espèces nouvelles pour le Burkina. Quelques données ponctuelles existent pour des stations situées plus en aval sur le Kou et le Mouhoun. Elles concernent les espèces suivantes : $C$. copiosa, $C$. digitata, C. falcifera, C. lesnei, A. senegalensis, $A$. dispar., C. mali et $E$. ulmeri ; leur présence confirme les résultats exposés au paragraphe précédent.

En revanche, la faune des hauts-cours du Mouhoun, du Kou, de la Léraba et de la Comoé mérite quelques commentaires.

Les résultats sont récapitulés dans le tableau 2. La taille de ce tableau est en elle-même un élément 
remarquable. Il comporte deux fois plus d'espèces pour un échantillonnage moins intensif. La zone étudiée présente donc une richesse spécifique supérieure à celle des régions voisines. Toutefois, l'élément le plus inattendu n'est pas le nombre, mais l'origine de ces espèces. Nous pouvons schématiquement les classer en trois groupes :

1) Un premier groupe comprend des espèces savanicoles qui n'avaient pas encore été signalées au Burkina. Hydroptila mariatheresae Gibon 1987,

Tableau 2. Trichoptères (part.) de la région de Bobo-Dioulasso (voir la localisation des stations Fig. 45).

Table 2. Trichoptera of the area of Bobo-Dioulasso (site localisation Fig. 45).

\begin{tabular}{|l|c|c|c|c|c|c|c|c|c|}
\hline & a & b & c & d & e & $\mathbf{f}$ & $\mathbf{g}$ & $\mathbf{h}$ & $\mathbf{i}$ \\
\hline Cheumatopsyche lesnei & & & + & & + & & & & \\
\hline Cheumatopsyche falcifera & + & + & & & + & & & & + \\
\hline Cheumatopsyche digitata & + & + & + & + & + & & + & + & \\
\hline Cheumatopsyche sexfasciata & + & & & & & & + & & \\
\hline Cheumatopsyche afra & + & + & & & & + & + & & \\
\hline Cheumatopsyche tournii & + & & & & & & + & & \\
\hline Cheumatopsyche macentae & & & & & & + & & + & \\
\hline Protomacronema barnardi & & & + & + & & & & & \\
\hline Protomacronema splendens & & & & & & + & + & & \\
\hline Leptonema aberrans & + & & & & & & & & \\
\hline Macrostemum inscriptum & & & & + & & + & & + & + \\
\hline Chimarra prodhoni & & & + & & & & & & \\
\hline Chimarra dybowskina & & + & & & & & & & \\
\hline Chimarra obala & + & & & & & & & & \\
\hline Chimarra occidentalis & & & & & & & + & & \\
\hline Ecnomus dinderessoi & & & & & + & & & & \\
\hline Ecnomus foliatus curvatus & & & + & + & & & & & \\
\hline Ecnomus similis & + & + & & & & & & & \\
\hline Ecnomus aberrans & & + & & & & & & & \\
\hline Ecnomus niouniouroui & + & + & & & & & & & + \\
\hline Hydroptila hirra & & & + & & & & & & \\
\hline Hydroptila mariatheresae & & & + & & & & & & + \\
\hline Hydroptila maoae & + & & & & & & & & \\
\hline Hydroptila judithae & + & & & & & & & & \\
\hline Catoxyethira veruta sept. & & + & & & & & & & \\
\hline Catoxyethira pinheyi & + & & & + & & & & & + \\
\hline Catoxyethira graboensis & & & & + & + & & & & \\
\hline Oxyethira cf. minima & & & & + & + & & & & \\
\hline Oxyethira burkina & & & & & & + & & & \\
\hline Oxyethira tenei & & & & + & & & & & \\
\hline Ugandatrichia atakpamensis & + & & & & & & & & \\
\hline Ugandatrichia lerabae & + & & & & & & & & \\
\hline
\end{tabular}


Catoxyethira veruta, Oxyethira cf. minima Gibon, 1987, Ecnomus aberrans Gibon, 1982, E. niouniouroui Gibon, 1992, E. foliatus curvatus Gibon, 1992, et Protomacronema barnardi Statzner et Gibon, 1985. D'après leurs répartitions géographiques et leurs exigences écologiques (outre les références déjà citées pour les Hydropsychidae et les Philopotamidae, on pourra consulter Gibon 1987 pour les Hydroptilidae et Gibon 1992 pour les Ecnomidae), leur présence à cette latitude et dans ces conditions n'est pas surprenante. En revanche, il est probable que ces espèces évitent les conditions extrêmes des zones soudaniennes et sahéliennes (assèchements prolongés, charges élevées en suspension, forte variabilité hydrologique), que l'on rencontre sur la majorité des cours d'eau du Burkina.

2) Un deuxième groupre comprend des espèces forestières ou de transition forêt/savane. Cheumatopsyche sexfasciata (Ulmer), 1904 en est un bon exemple, sa distribution est représentée sur la figure 47. La comparaison sur cette même carte de $C$. sexfasciata et de $C$. lesnei est démonstrative. L'une comme l'autre colonise une large gamme de cours d'eau ; le premier en forêt et savanes guinéennes, le second en savanes sahélo-soudaniennes. La disposition latitudinale des principales zones de végétation de l'Afrique occidentale induit une quasiexclusion géographique de ces deux espèces. La présence de $C$. sexfasciata sur les cours supérieurs du Mouhoun et de la Léraba constitue la seule exception connue à ce partage. Nous pouvons inclure également dans ce groupe : Chimarra prodhoni Gibon, 1985, caractéristique des zones de transition forêt/savane, Chimarra occidentalis Gibon, 1985, au caractère forestier plus marqué, Economus similis Mosely, 1932, Catoxyethira graboensis Gibon, 1985 et $C$. pinheyi Kimmins, 1958. La figure 49 indique, par exemple, la répartition connue de $C$. pinheyi en Afrique occidentale. Elle comprend la dorsale guinéenne (une série de massifs qui s'étendent depuis le sud du Fouta Djalon jusqu'au Mont Tonkoui en Côte d'Ivoire et sont relativement bien arrosés) ainsi que les forêts, sud-ouest ivoirien. La présence de l'espèce sur le Kou élargit son aire géographique, tout en révélant sur ce bief des conditions faunistiques particulières. Le cas de Macrostemum inscriptum (Walker), 1852 est particulier ; il appartient probablement à cette catégorie, comme le laisse supposer sa répartition dans le bassin du Niger (Fig. 46). En revanche, il est sporadique dans le bassin du
Bandama où sa répartition a pu être très perturbée par les traitements insecticides (Statzner \& Gibon 1984) et ce phénomène a pu également avoir lieu au Burkina.

3) Un troisième groupe comprend des espèces que nous qualifierons de " montagnardes », bien qu'en Afrique occidentale ce terme soit un peu présomptueux. La distribution de nombreuses espèces est en effet liée à celle des petits massifs de la région. $P$. splendens Ulmer, 1905 en est un exemple caractéristique (Fig. 46). Sa répartition en Afrique occidentale comprend : le Fouta Djalon, les massifs du Ziama et du Fon (dorsale guinéenne) et le Mont Nimba. Il s'agit d'une espèce rare et strictement localisée aux zones montagneuses. Le plateau de Bobo-Dioulasso constitue la limite inférieure de sa distribution altitudinale. Autre espèce caractéristique des zones de relief plus accentué, Cheumatopsyche afra (Mosely), 1935 (Fig. 48) se rencontre, outre les localités précédemment citées, en Côte d'Ivoire sur le Mont Tonkoui, au Togo dans la région du plateau de Danyi et au Mali dans les Monts Mandingues. Cheumatopsyche tournii n. sp. (Fig. 48), C. macentae n. sp., Hydroptila judithae Gibon, 1987, $H$. maoae n. sp. (Fig. 49), Oxyethira tenei n. sp. (Fig. 50), O. burkina n. sp., Ugandatrichia atakpamensis Gibon, 1987 (Fig. 50) et $U$. lerabae n. sp. sont également à inclure dans ce groupe.

Les quatre biefs présentent donc, sur une distance assez courte (quelques dizaines de kilomètres depuis leur source), une faune riche et diversifiée, composée d'espèces avec des affinités forestières ou montagnardes. Plusieurs explications peuvent être retenues. L'altitude est relativement élevée (stations situées entre 340 et $520 \mathrm{~m}$, soit une centaine de mètres au-dessus des cours supérieurs du Nzi/Bandama). Une seconde particularité de ces cours d'eau est la continuité de l'écoulement au cours du cycle hydrologique annuel, ce qui est rare à cette latitude (voir, par exemple, Statzner 1984). Enfin la végétation est souvent protégée et l'impact agricole réduit, soit par l'existence de forêts classées (f.c. du Kou, f.c. de Dinderesso) soit par l'escarpement du relief (vers Tourni et Karfiguéla, par exemple). Ces trois facteurs créent un ensemble de conditions (températures plus faibles, transparence plus élevée) qui atténuent le caractère « savane " du cours d'eau. Sans que l'on puisse, pour l'instant, disposer d'une hiérarchisation, ou d'une quantification du 
phénomène, une étude faunistique, même partielle, en permet une spectaculaire mise en évidence.

\section{Discussion et perspectives}

Dans l'état actuel des connaissances, il n'est pas possible de généraliser ce phénomène à l'ensemble de la faune benthique. Toutefois, il est déjà connu pour deux autres groupes. Nous l'avons évoqué récemment pour le genre Trichosetodes, avec la présence sur le Kou de T. pauli Gibon, 1991 ; mais surtout, il avait été signalé chez les Simulies (Diptera, Dimuliidae) vectrices d'Onchocerca volvulus Leuckart 1893. Il s'agit d'un groupe restreint, mais les données sur la répartition géographique des espèces reposent sur un réseau d'échantillonage d'une densité exceptionnelle (Quillévéré \& Pendriez 1975). Les deux espèces caractéristiques des savanes sont Simulium damnosum s.s. et surtout $S$. sirbanum Vajime \& Dunbar, 1975. Cependant, S. squamosum Vajime \& Dunbar, 1975, une espèce « forestière » ayant de fortes affinités pour les petites rivières (par opposition à S. soubrense Vajime \& Dunbar, 1975 ou S. sanctipauli Vajime \& Dunbar, 1975) a été observée dans des « zones de savane ombragée relativement humide » (Quillévéré 1979). Ces zones sont l'Atakora au Bénin et son prolongement au Togo, ainsi que la région de Bobo-Dioulasso au Burkina. Etant donné la durée de vie des adultes et leur capacité de dispersion, bien supérieures à celles des Trichopères, ces observations étaient supposées consécutives à de nouvelles invasions annuelles accompagnant la remontée du Front Intertropical vers la savane, au début de la saison des pluies. Quelle qu'en soit l'origine, il s'agit, chez ces Simulies, d'un phénomène identique à celui décrit ici chez les principales familles de Trichoptères. Cette coïncidence laisse supposer qu'il est bien plus général, et c'est l'une des voies pour poursuivre ce travail.

Une autre question soulevée par ces observations est leur généralisation géographique. Un autre cas est connu, bien qu'il n'ait pas fait l'objet d'une publication particulière, il s'agit du Woyowayanko, un petit affluent du Niger. Il prend sa source dans les Monts Mandingues et se jette dans le Niger, une dizaine de kilomètres plus loin, au niveau des quartiers Ouest de Bamako. Son cours supérieur présente une faune de Trichoptères très différente de celle de deux rivières voisines, du Niger lui-même ou du cours supérieur du Baoulé/Sénégal (qui prend également sa source dans les Monts Mandingues, mais sur le versant nord). On y rencontre Macrostemum inscriptum (Fig. 46), Cheumatopsyche afra (Fig. 48), Chimarra dybowskina Navas, 1933, Chimarra remyi Gibon, sous presse (dont la seule autre station connue est dans le Fouta-Djalon) et Chimarra mandingorum Gibon, sous presse (qui n'est connu que de ce site, mais devrait être présent également sur le Fouta Djalon). C. remyi et C. mandingorum sont, par ailleurs, phylogénétiquement éloignés de $C$. minima, $C$. sassandrae et $C$. callasae Gibon, 1982, les trois Chimarra savanicoles qui, le Woyowayanko excepté, sont les seules espèces connues au Mali. Il existe donc sur le cours supérieur du Woyowayanko une situation similaire, quoique moins marquée, à celle décrite dans la région de Bobo-Dioulasso. Par ailleurs, les indications fournies par Quillévéré (1979) laissent supposer qu'une étude faunistique du massif de l'Atakora (Bénin) permettrait des observations identiques. Enfin, la zone des cours supérieurs du Sankarani, du Baoulé/Niger et du Sassandra, rivières de la région d'Odiénné en Côte d'Ivoire, devrait être encore plus riche, car elle est située à des niveaux d'altitude égaux ou supérieurs et bénéficie d'une pluviométrie plus élevée.

Nous disposons de suffisamment d'indices pour affirmer que l'homogénéité faunistique des fleuves et rivières de savane est effectivement une conséquence des faibles gradients d'altitude. Elle disparaît dès que l'on atteint les environs de $400 \mathrm{~m}$. Nous retrouvons alors un schéma de zonation longitudinale plus conforme aux théories classiques. En revanche, dans de telles situations, la distribution des espèces de la transition forêt/savane est atypique. Elles colonisent les cours supérieurs (situés dans les zones d'altitude) ainsi que les cours inférieurs lorsque le fleuve pénètre en zone forestière, mais elles sont absentes des zones moyennes du bassin situées en savane (cas de Cheumatopsyche sexfasciata dans le bassin de la Comoé).

\section{Annexes taxinomiques}

\subsection{Cheumatopsyche}

A propos des Cheumatopsyche, Botosaneanu (1992) écrit « Chaos still reigns in the systematic knowledge of the genus in some parts of the world, and distinguishing related species from one another is often an arduous task, particularly in view of the 
very simple genitalia ". Cela décrit assez bien la situation de beaucoup d'espèces afrotropicales. La simplicité des génitalia et leur homogénéité à l'intérieur du genre sont effectivement deux difficultés majeures, auxquelles il faut ajouter le fait que le dixième segment abdominal ne soit pas une pièce dure mais un élément aisément déformable à la suite de manipulations ou de dessiccation. En outre, de faibles différences dans l'orientation des genitalia au cours du montage peuvent modifier de manière notable le schéma qui sera publié par la suite. Enfin certaines espèces ont une vaste distribution, ou présentent des populations isolées les unes des autres, ce qui induit des variations morphologiques difficiles à interpréter, dans la mesure où les études géographiques sont exceptionnelles. Les synthèses comparatives ont été peu nombreuses et la confusion règne donc effectivement sur de nombreux points. En Afrique occidentale, les premières tentatives de Gibbs (1973) au Ghana, mais surtout le travail de Statzner (1984) en Côte d'Ivoire, fournissent une base qui permet progressivement l'élaboration de la faune régionale.

\section{Cheumatopsyche tournii n. sp.}

Matériel : holotype mâle capturé sur la haute Léraba (bassin de la Comoé, région de Banfora, Burkina Faso), à proximité du village de Tourni le 23.1.1993, rec. F.-M. G. et B. C., un paratype mâle capturé sur le haut Mouhoun (bassin des Voltas, région de Bobo-Dioulasso, Burkina Faso), à proximité du village de Kourinion le 20.2.1991, rec. W. G.. Etymologie : le village de Tourni sur la haute Léraba.

Description : Fig. 1 à 5 , Fig. 12. La coloration des ailes est brun pâle, avec quelques rares taches plus claires dans la zone dorso-distale. Ce seul caractère suffirait à distinguer $C$. tournii de $C$. digitata dont les ailes sont brun sombre et présentent de nombreuses taches très contrastées. Au dixième segment abdominal, les appendices digitiformes situés à chaque bord latéro-distal se composent d'une branche dorsale nettement allongée et d'une courte branche ventrale, qui évoque plutôt un bouton ou une bosse. Cette structure est très particulière, elle a été décrite chez $C$. digitata, la distinction des deux espèces se fera à partir des formes respectives des deux branches. Chez $C$. tournii, la branche supérieure est plus longue, plus nettement marquée ; son insertion se situe (vue latérale) au niveau de la verrue spinifère. Chez $C$. digitata, l'insertion de la branche supérieure est plus distale (Fig. 6 et 8). La branche ventrale est moins apparente, elle $n$ 'est pas en forme de bosse mais a tendance à se développer perpendiculairement au plan dorso-ventral, son insertion est moins externe et plus dorsale, située au-dessus de la bande sombre et sclérotisée qui marque la partie inférieure du dixième segment. Chez $C$. digitata, la branche ventrale offre une sémytrie axiale, sa base est située dans le prolongement de la bande sclérotisée. Enfin (vue dorsale), la verrue sétifère est plus saillante. Les appendices inférieures permettent une distinction aisée des deux espèces. Chez C. tournii, la partie distale du premier article est fortement épaissie, le deuxième article est mince, il présente une courbure nette (Fig. 3). Chez $C$. digitata, l'extrémité du premier article forme un réceptacle où s'insère un deuxième article très élargi et de forme caractéristique (Fig. 9).

Taille: la longueur de l'aile antérieure varie de 5,5 à $6.2 \mathrm{~mm}$, celle de l'aile postérieure de 4,3 à $4,8 \mathrm{~mm}$.

Répartition et variations géographiques : seuls les exemplaires du Burkina ont été inclus dans le matériel type. C. tournii a également été capturé en Guinée sur les bassins du Sénégal et du Rio Corubal, dans le Fouta Djalon (Fig. 48). Il existe également au Cameroun : sur l'Assamba à Nkomeyo aux environs de Yaoundé (bassin de la Sanaga), sur le plateau de l'Adamaoua dans la région de Ngaoundéré et sur le Mayo Louti en amont de Mokolo (bassin du Logone). Si les spécimens guinéens ne présentent de différence morphologique notable avec ceux du Burkina, les exemplaires capturés au Cameroun se caractérisent par quelques variations au niveau des appendices digitiformes du dixième segment abdominal. La branche supérieure s'élargit vers l'extrémité distale, ce qui lui donne une légère forme en massue (Fig. 4 et 5), la branche inférieure est plus développée et plus saillante, elle accuse une légère orientation vers l'axe central. $C$. tournii ayant une distribution limitée à des zones d'altitude, les différentes populations sont isolées et il n'est pas étonnant de voir apparaître des différenciations morphologiques, auxquelles il serait prématuré de donner un statut taxinomique.

Espèces voisines : la division des appendices digitiformes du dixième segment abdominal en deux parties est rare chez les Cheumatopsyche, elle caractérise bien C. digitata et $C$. tournii. On l'observe chez deux autres espèces. La première, C. leloupi Jacquemart 1957 a été considérée comme un synonyme de $C$. digitata par de nombreux auteurs dont Gibbs et Statzner ; la coloration de l'aile et la forme des appendices inférieurs sont typiques de $C$. digitata. La seconde, C. antoniensis Malicky 1982 est une forme vicariante de $C$, digitata, isolée aux lles du Cap Vert.

\section{Cheumatopsyche macentae n. sp.}

Matériel : holotype mâle capturé sur la Loffa à Macenta (Guinée), le 20.2.1987, rec. J.-F. Agnèse ; un paratype mâle sur le haut Mouhoun à Orodara le 21.2.1991, rec. W. G. ; un paratype mâle sur la Dienkoa (affluent du haut Mouhoun, Burkina Faso) au niveau de l'axe routier BoboDioulasso / Sikasso, le 25.1.1993, rec. F.-M. G. et B.C. Etymologie : Macenta, ville de Guinée. 
Description. Fig. 10, 13 et 14. C. macentae appartient à un groupe d'espèces remarquablement homogène quant aux caractères morphologiques. En Afrique occidentale, il comprend au moins : C. falcifera, C. afra, C. akana Statzner, 1984 et $C$. gibbsi Statzner, 1984. La structure des genitalia est identique chez toutes ces espèces ; ce sont les proportions des divers éléments du dixième segment abdominal qui permettent la diagnose. Les ailes offrent le même aspect que celles de $C$. falcifera, colorées d'un brun moyen moucheté de taches plus ou moins rondes d'un brun plus pâle (voir Statzner 1984). A l'extrémité du dixième segment abdominal, les appendices digitiformes sont gros et épais, proportionnellement plus développés que chez $C$. falcifera mais moins allongés que chez $C$. gibbsi ou C. akana. Sur la vue latérale, ils sont à peine dirigés dorsalement et, sur la vue dorsale, légèrement dirigés vers l'axe central. L'extrémité du bord centro-dorsal est (vue latérale) situé antérieurement à l'extrémité distale des appendices digitiformes ; ceci est un caractère du groupe falcifera et le distingue d'autres groupes où ce bord centro-distal se situe au niveau de l'extrémité des appendices digitiformes voire un peu en arrière comme chez $C$. lestoni Gibbs, 1973. En vue dorsale ce bord est droit voire très légèrement arrondi, et non pas saillant. Les appendices inférieurs sont minces et élancés ; l'élargissement distal du premier article est présent mais peu marqué. Le deuxième article est large et peu arqué. Ces deux caractères distinguent $C$. macentae de $C$. toumii (Fig. 3) et $C$. afra (Fig. 11) où l'on observe à la fois un fort épaississement de la partie terminale du premier article et la courbure prononcée d'un second article mince et élancé.

Taille : la longueur de l'aile antérieure varie de 5,6 à $5,8 \mathrm{~mm}$, celle de l'aile postérieure de 4,1 à $4,5 \mathrm{~mm}$.

Espèces voisines : chez $C$. diminuta Walker 1953 et C. urema Mosely 1936 les appendices digitiformes sont bien plus longs ; chez C. plutonis Banks 1913, le bord centro-distal présente une profonde ouverture en forme de V, enfin chez C. thomasseti Ulmer 1931 les appendices digitiformes sont plus écartés.

\section{Cheumatopsyche afra Mosely, 1935}

C. afra est une espèce répandue sur tout le continent au sud du Sahara. En Afrique occidentale, elle est disséminée en populations isolées sur les massifs montagneux (Fig. 48). Nous avons présenté (Fig. 15 et 16) des vues latérales et dorsales des génitalia mâles de façon à pouvoir les comparer à $C$. tournii et $C$. macentae.

\subsection{Ecnomus}

\section{Remarques préliminaires sur l'armature génitale des Ecnomus}

Le dixième segment abdominal est composé de deux ensembles symétriques par rapport au plan dorso-ventral. Chacun comprend généralement un lobe lamellaire (souvent considéré comme l'appendice supérieur), ainsi qu'une branche interne plus fortement sclérotisée, parfois appelée « upper penis cover » et insérée à la base du lobe lamellaire, côté interne. Chez beaucoup d'espècs, l'on observe sur la face interne du lobe lamellaire, un processus (que nous nommerons processus interne) de forme peu distincte, globuleuse à digitiforme, portant quelques fortes soies à son extrémité.

\section{Ecnomus wendmanegrei n. sp}

Matériel : holotype et un paratype mâles capturés sur la Léraba (bassin de la Comoé, Côte d'Ivoire) au pont frontière, sur l'axe routier Bobo-Dioulasso / Ferkéssédougou, le 27.4.1993, rec. F.-M. G. et M. Bihoum ; un paratype mâle sur le Mouhoun à Toukoro (Burkina Faso) le 12.12.1990, rec W. G.. Etymologie : cette espèce est dédiée à Mr. Régis Wendmanégré Yaméogo.

Description : Fig. 18 à 20. La formule calcarienne est $2 / 4 / 4$, il n'y a pas d'éperon préapical aux pattes du premier segment thoracique. Cette absence est remarquable. Dans la zone afrotropicale, la forme est habituellement 3/4/4 sauf chez E. bifurcatus (Mosely), 1935 (qui présentent d'autres particularités morphologiques, comme la division des appendices inférieurs) et chez les espèces du groupe natalensis (Kimmins 1957). Or par les caractères de l'armature génitale, $E$. wendmanegrei se distingue nettement du groupe natalensis comme d'E. bifurcatus. Il représente une troisième lignée afrotropicale ayant perdu l'éperon préapical des pattes antérieures.

Genitalia : le lobe lamellaire est arrondi à l'extrémité, laquelle est décalée vers le bord ventral. Les épines internes sont minces et situées en retrait de l'extrémité. Le processus interne est globuleux et peu développé. La branche interne est caractéristique ; en vue latérale, elle est courbée, très large à la base et régulièrement amincie vers l'apex ; en vue dorsale, elle est large, cet élargissement se produit perpendiculairement au plan dorso-ventral. Dirigée distalement à la base, elle présente ensuite un coude brusque à convexité externe et se termine par trois soies alignées sur le bord distal. Les appendices inférieurs sont courts, grossièrement ovales en vue latérale. Leur extrémité distale est située à peu près au même niveau que celle du dixième segment. Il n'y a pas de branche digitiforme disto-interne comme dans le groupe natalensis. L'appareil phallique est massif, dépourvu de paramères, la sclérotisation accentuée de la partie inférieure offre une ligne ventrale heurtée (distincte de la ligne sinueuse présente chez la plupart des espèces) et isole un bec distal de forme caractéristique.

Taille: la longueur de l'aile antérieure est de $3,2 \mathrm{~mm}$, celle de l'aile postérieure de $2,6 \mathrm{~mm}$.

\section{Ecnomus dinderessoi n. sp.}

Matériel : holotype mâle capturé sur le Mbé (affluent du Nzi, bassin du Bandama, Côte d'Ivoire) dans la région 
de Satama-Sokoura le 21.12.1982, rec. F.-M. G. et M. Bihoum ; un paratype mâle sur l'Oti (bassin des Voltas, Ghana) à Sabari de 16.11.1985, rec. J. Shorscher ; un paratype mâle sur le Kou (affluent du Mouhoun, bassin des Voltas, région de Bobo-Dioulasso, Burkina Faso) à Dinderesso, le 11.12.1990, rec. W.G. Etymologie : Dindérésso, village des environs de Bobo-Dioulasso.

Description : Fig. 21 et 22 . La formule calcarienne est 3/4/4. La forme (vue latérale) du lobe lamellaire est caractéristique : grossièrement trapézoïdale avec l'anglo dorsodistal déformé en un prolongement digitiforme court portant un groupe de grosses soies interne à son extrémité. Le processus interne est allongé et présente une ébauche de ramification. La branche interne est longue, cylindrique, spiniforme vers l'extrémité distale ; elle est d'abord dirigée ventralement puis distalement, le coude est assez accentué. L'appendice inférieur est massif et grossièrement triangulaire en vue latérale ; son extrémité distale est située au-delà de celle du dixième segment. Cette forme est commune dans le genre Ecnomus; l'importance relative de l'appendice par rapport à l'ensemble de l'armature génitale est, en revanche, spécifique. L'appareil phallique présente une structure classique : la partie sclérotisée, large à la base, est progressivement réduite au bord ventral et prolongée distalement en un bec courbé ventralement.

Taille : la longueur de l'aile antérieure varie de 3,5 à $3,7 \mathrm{~mm}$, celle de l'aile postérieure de 2,8 à $3,1 \mathrm{~mm}$.

Espèces voisines : $E$. dinderessoi est très proche d' $E$. dispar Kimmins 1957. La structure des armatures génitales est identique, mais chaque élément présente une forme différente. Chez $E$. dispar (Fig. 23), les appendices inférieurs sont proportionnellement plus développés et plus longs, l'extrémité distale de l'édéage est légèrement moins courbée, arrondie et non pointue. Au dixième segment abdominal, le coude de la branche interne est moins accusé. C'est la forme du lobe lamellaire qui constitue le caractère le plus distinctif ; il est, chez dispar, grossièrement triangulaire et le sommet (distal) est déformé en un court prolongement arrondi. $E$. dispar a été décrite du bassin du Nil (Ouganda) et l'exemplaire que nous figurons ici a été capturé dans le nord du Cameroun (sur le Mayo Boki au niveau de l'axe routier Ngaoundéré/Garoua, bassin de la Bénoué). Nous avons noté également sa présence sur le Niger dans la région de Bamako (Mali). Sa répartition, à priori plutôt sahélienne, est vaste, bien qu'elle ne semble pas être très abondante. Ces éléments indiquent qu' $E$. dinderesso $i$ et $E$. dispar sont deux espèces distinctes et non deux formes géographiques vicariantes. $E$. ferrantei Ulmer 1963 est également très proche de ces deux formes, plus encore d' $E$. dispar que d' $E$. dinderessoi. Cette parenté est remarquable car $E$. ferrantei est une espèce nord-africaine, provenant du bassin du Nil (Egypte). Il s'agit d'un cas d'affinité entre les faunes afrotropicale et méditerranéenne. Ces derniers ne sont pas nombreux et, dans le genre Ecnomus, un premier cas a déjà été signalé celui d'E. kunenensis Barnard 1934 et d'E. galilaeus Tjeder 1946, le premier largement répandu en Afrique subsaharienne, le second connu du Levant. Dans chaque cas, l'origine et les affinités sont afrotropicales.

\section{Ecnomus ulmeri Mosely, 1932}

E. ulmeri est l'espèce la plus largement répandue en Afrique occidentale. Les exemplaires du Mouhoun offrent peu de variations par rapport au matériel type (provenant de Sierra Leone). On notera que l'extrémité de la branche interne du dixième segment abdominal est légèrement courbée dorsalement alors qu'elle reste droite dans la description originale. La distinction avec les autres espèces de la région ne pose aucun problème du fait des déformations latérales de la phallothèque (Fig. 17).

\subsection{Catoxyethira}

\section{Catoxyethira graboensis Gibon, 1985}

Tous les exemplaires examinés diffèrent de la description originale par la présence d'une épine noire supplémentaire au bord latéro-distal du huitième segment abdominal. Sur le type, ces épines sont au nombre de deux (de chaque côté) ; la première est insérée à l'angle dorsal, elle est un peu plus courte, la seconde est insérée ventralement (en vue latérale : à la hauteur des appendices inférieurs). Sur le matériel burkinabe (Fig. 24), on observe de légères modifications dans l'orientation de ces épines ; la dorsale est faiblement orientée ventralement, la ventrale est droite, non courbée à l'extrémité. Mais surtout, il existe une troisième épine insérée juste au-dessous de la ventrale et nettement plus courte (un peu moins de la moitié de la longueur de cette dernière).

\subsection{Ugandatrichia}

\section{Ugandatrichia lerabae n. sp.}

Matériel : holotype et cinq paratypes mâles capturés sur la haute Léraba (bassin de la Comoé, région de Banfora, Burkina Faso), à proximité du village de Tourni le 23.1.1993, rec. F.-M. G. et B. C.. Etymologie : la Léraba, affluent de la Comoé.

Description : Fig. 25 à $27 ; 30$ et 31 . U. lerabae est un hydroptilide de taille moyenne et de formule calcarienne $0 / 3 / 4$, il présente trois ocelles et est dépourvu de suture transverse au mésoscutellum. Ce mésoscutellum est un peu plus large que celui des autres Ugandatrichia (voir Fig. 30, et pour comparaison, Fig. 32 le mésoscutellum d'U. yameogoi Gibon, 1987) la forme est intermédiaire entre celle d'Ugandatrichia et celle d'Ithytrichia. En revanche le métascutellum présente une forme plus proche de celle du genre Ugandatrichia (Fig. 31 et 33). Nous avons classé cette espèce dans le genre Ugandatrichia, mais la distinction de ces deux genres mériterait un réexamen. 
D'une part, la similitude des structures génitales est remarquable, d'autre part la forme du mésoscutellum n'est peutêtre pas un caractère aussi discriminant qu'on ne le pensait (Marshall 1979).

Genitalia : le neuvième segment abdominal est massif ; il développe, de chaque côté, un lobe distal arrondi qui masque les autres pièces génitales. Ventro-distalement, il présente une profonde ouverture où s'insèrent les appendices inférieurs. Le dixième segment abdominal forme une masse indistincte au-dessus de l'appareil phallique. L'élément le plus caractéristique est constitué par la plaque sous-génitale qui est située sur deux niveaux. Dorsalement, elle est trilobée, deux lobes latéraux digitiformes et symétriques encadrent un élément central plus sclérotisé, dirigé distalement et de forme caractéristique (vue dorsale). Cet ensemble se prolonge ventralement par un élément sclérotisé profondément ouvert distalement et dont la forme en U (Fig. 25 et 26) est également spécifique. Les appendices inférieurs sont courts, larges mais assez peu épais. L'appareil phallique est typique du genre Ugandatrichia, il présente un titillateur spiralé court mais très apparent.

Taille : la longueur de l'aile antérieure varie de 2,0 à $2,1 \mathrm{~mm}$, celle de l'aile postérieure varie peu autour de $1,8 \mathrm{~mm}$.

Espèces voisines : par certains aspects $U$. lerabae est assez différent des Ugandatrichia connus en Afrique occidentale, en particulier la complexité de l'ensemble sousgénital (le terme « plaque » est ici impropre), qui évoque le genre Dhatrichia. On note également la forme des appendices inférieurs intermédiaire entre le type « longs et parallèles " (que l'on observe chez $U$. atakpamensis (Fig. 29) ou $U$. minor Mosely 1939 et le type « courts et disposés en $\mathrm{V}$ » que l'on observe chez $U$. rhodesiensis Scott 1976 ou $U$. nigra Mosely 1939.

\section{Ugandatrichia atakpamensis Gibon, 1987}

Cette espèce a été décrite du Togo, à partir de spécimens provenant des petits massifs situés au sud-ouest du pays, à la frontière ghanéenne. Depuis, sa présence a été constatée dans le Fouta Djalon (Guinée) et au Burkina Faso. Nous figurons (Fig. 28) une vue dorsale de la plaque sous-génitale qui offre de légères différences morphologiques avec le matériel togolais (la population du Fouta Djalon est très proche de celle du bassin de la Comoé). Ce phénomène doit être considéré comme un indice de l'isolement des populations, mais ne justifie pas la création d'une unité taxinomique originale.

\subsection{Hydroptila}

\section{Hydroptila maoae n. sp.}

Matériel : holotype et trois paratypes mâles capturés sur la haute Léraba (bassin de la Comoé, région de Banfora, Burkina Faso), à proximité du village de Tourni le
23.1.1993, rec. F.-M. G. et B. C.. Etymologie : Mme Marie-Odile Barbe.

Description : Fig. 34 à 37 . Il existe une petite expansion ventro-médiane distale sur le septième sternite abdominal. Le neuvième sternite présente de longs prolongements antérieurs séparés par une profonde incision en $\mathrm{U}$ qui atteint la base des appendices inférieurs. Chaque bord distal de ce segment forme, à mi-hauteur, un prolongement digitiforme, plus mince en vue ventrale. Ces « projections distales $»$ du neuvième segment ne sont pas rares chez les Hydroptila. Le dixième segment forme une masse peu distincte en toit au-dessus de l'appareil phallique, son bord distal n'est pas incisé en V comme chez d'autres espèces (H. sparsa Curtis, 1834 ou angustata Mosely, 1939, par exemple), il est droit, voire légèrement convexe. Les appendices inférieurs sont droits, en forme de massue (vue latérale), il existe un bec distal sclérotisé dirigé vers l'extérieur, ainsi qu'une petite bosse sclérotisée située à peu près aux deux-tiers de la longueur. Ils sont dépourvus de branche supérieure. La plaque sous-génitale se présente sous la forme en Y caractéristique (la base du Y est distale) ; sur la vue latérale, elle apparaît comme une bande sombre, prenant naissance à la base du dixième segment et dirigée ventro-distalement. L'appareil phallique est remarquable par le curieux enroulement terminal du filament spiral et par sa longueur exceptionnelle.

Taille : la longueur de l'aile antérieure varie de 2,0 à $2,1 \mathrm{~mm}$, celle de l'aile postérieure de 1,8 à $1,9 \mathrm{~mm}$.

Répartition géographique : le matériel type a été restreint aux spécimens provenant de Burkina; mais $H$. maoae a été capturée à plusieurs reprises dans le massif du Fouta-Djalon et sur la dorsale guinéenne (figure 49). Elle est présente sur les cours supérieurs du Rio Corubal (région de Télimélé), du Bafing (région de Dalaba), du Tinkisso (Dabola), du Milo (massif du Going), du Diani (Massif du Fon) et de la Férédougouba (massif du Tétini).

Espèces voisines: $H$. maoae appartient au groupe pulchricornis caractérisé par la plaque sous-génitale en $Y$, elle est proche d' $H$. aegyptia Ulmer 1963, elle présente une structure génitale semblable mais s'en distingue immédiatement par la longueur du neuvième sternite, celle de l'appareil phallique ou la forme du dixième segment.

\subsection{Oxyethira}

\section{Oxyethira burkina n. sp. (sp. H)}

Matériel : hologype mâle capturé sur le haut Mouhoun à Orodara en 2.1991 , rec. W. G. ; deux paratypes mâles sur un petit affluent du Rio Corubal au niveau de l'axe routier Gaoual/Télimélé (Guinée) le 27.1.1987, rec. F.M. G.

Description : Fig. 38 à 41 . O. burkina est remarquable par sa formule calcarienne : $0 / 2 / 4$ (l'éperon préapical des P2 manque) et par la fusion des radiales 4 et 5 aux ailes 
antérieures. Ces deux caractères permettent d'inclure $O$. burkina dans le sous-genre Dampfitrichia (Kelley 1984). Cette appartenance est confirmée par la structure génitale ; on remarquera, cependant, l'absence de processus dorso-latéraux au huitième segment abdominal.

Génitalia : le huitième segment abdominal forme un anneau autour de l'armature génitale. Cet anneau est nettement moins large dorsalement, il ne présente pas d'échancrure ventrale (à peine une faible concavité distale). On n'y observe aucune déformation particulière, ni aucune épine distale. Le neuvième segment présente un allongement ventral antérieur, net mais peu important et sans aucune échancrure médiane. Il présente également un allongement ventral postérieur dû à la fusion avec les appendices inférieurs. La vue ventrale de l'ensemble permet d'observer un rétrécissement puis une concavité distale qui sépare deux projections arrondies et symétriques. L'ensemble est très semblable à $O$. cf. minima, on notera que le bord antérieur est légèrement convexe àlors qu'il est plutôt concave chez minima. La plaque sous-génitale est large et épaisse à sa base puis forme une plaque de forme grossièrement trapézoïdale sans invagination du bord distal. Le dixième segment est réduit à une masse membraneuse peu distincte, qui est représentée sur la figure 38, mais n'est pratiquement pas visible après éclaircissement à la potasse. La structure de l'appareil phallique est simple, on n'y observe pas de titillateur spiralé. L'extrémité distale est large et forme une séries de petites dents caractéristiques.

Taille : la longueur de l'aile antérieure varie de 1,7 à $1,8 \mathrm{~mm}$, celle de l'aile postérieure de 1,5 à $1,6 \mathrm{~mm}$.

Espèces voisines: les trois espèces africaines $O$. cf minima, $O$. burkina et $O$. tenei sont proches ; elles peuvent néanmoins se distinguer aisément par l'apex de l'édéage qui présente souvent dans le genre Oxyethira des formations spécifiques.

\section{Oxyethira tenei n. sp. (sp. D)}

Matériel : holotype mâle capturé sur le Téné (bassin du Sénégal, Fouta-Djalon, Guinée) à Kala, le 24.1.1987, rec. F.-M. G. ; un paratype mâle sur le Kou (affluent du Mouhoun, bassin des Voltas, région de Bobo-Dioulasso, Burkina Faso) à Dinderesso le 22.1.1993, rec. F.-M. G. et B. C.. Etymologie : le Téné, affluent du Bafing.

Description : Fig. 42 à 44 . O. tenei présente la formule calcarienne $0 / 2 / 4$ et la fusion des radiales 4 et 5 aux ailes antérieures (ce dernier caractère est peu visible), elle appartient donc au sous-genre Dampfitrichia, mais, comme $O$. burkina est dépourvue de processus dorso-latéraux au huitième segment abdominal.

Génitalia : le huitième segment abdominal forme un anneau autour de l'armature génitale. Cet anneau, moins large dorsalement, ne présente pas d'échancrure ventrale.
On n'y observe aucune déformation particulière, ni aucune épine distale. Le neuvième segment présente un allongement ventral antérieur plus développé que chez $O$. burkina et sans aucune échancrure médiane. Il présente également un allongement ventral postérieur dû à la fusion avec les appendices inférieurs. Cet allongement est plus développé et plus long ; il n'y a pas de rétrécissement en épaisseur (vue dorsale), ce qui se traduit par une concavité du bord distal beaucoup plus profonde. Le bord distal de la plaque sous génitale, qui était droit chez $O$. bur$k i n a$, offre ici une profonde ouverture en $\mathrm{U}$. Chacun des processus digitiformes ainsi formés porte une petite bosse ventrale. L'appareil phallique est remarquable par l'individualisation distale du canal spermatique ; parallèlement la phallothèque forme un lobe digitiforme qui porte deux expansions spiniformes courbes et dissymétriques (l'une est un peu plus longue et présente une inversion de courbure).

Taille : la longueur de l'aile antérieure est de 1,7 mm, celle de l'aile postérieure de $1,5 \mathrm{~mm}$.

Répartition géographique (Fig. 50) : O. tenei a été capturée dans le massif du Fouta-Djalon sur les cours supérieurs de plusieurs bassins : le Rio Corubal (région de Télimélé), la Kolenté, le Konkouré (régions de Kouri et de Linsan) et le Bafing (régions de Kala et de Dalaba). Sa présence sur la dorsale guinéenne n'a été observée que sur le Mafou (axe routier Faranah/Kissidougou), elle y est probablement bien plus répandue.

\section{Dépôt du matériel}

Il fait actuellement partic de la collection du premier auteur, à l'antenne Orstom auprès du laboratoire d'Ichtyologie générale et appliquée du Museum National d'Histoire Naturelle (Paris). Il sera déposé, ultérieurement, au Laboratoire d'Entomologie de cette institution.

\section{Travaux cités}

Botosaneanu L. 1979. - Quinze années de recherches sur la zonation des cours d'eau : 1963-1978. Revue commentée de la bibliographie et observations personnelles. Bijdr. tot. Dierkunde, 19 (1) : 109-134.

Botosaneanu L. 1992. - Fauna Palestina, Insecta VI - Trichoptera of the Levant - Imagines. The Israel Academy of Sciences and Humanities. 293 p.

Elouard J.-M.. 1983. — Impact d'un insecticide organophosphoré (le téméphos) sur les entomocénoses associées aux stades préimaginaux du complexe Simulium damnosum (Diptear : Simuliidae). Thèse Doctorat d'Etat, Université Paris XI, centre d'Orsay : $576 \mathrm{p}$.

Gibbs D.G. 1973. - The Trichoptera of Ghana. Dt. ent. Z., 20 $(4,5): 363-424$.

Gibon F.-M. 1985. - Recherches sur les Trichoptères d'Afrique de l'Ouest. II - Stactobiini (Hydroptilidae) de Côte d'Ivoire. Revue fr. Ent., (N.S.), 7 (4) : 149-155.

Gibon F.-M. 1985. - Recherches sur les Trichoptères d'Afrique Occidentale. 3-Philopotamidae de Côte d'Ivoire. Rev. Hydrobiol. trop., 18 (1) : 23-30. 
Gibon F.-M. 1987. - Recherches sur les Trichoptères d'Afrique Occidentale. 8-Hydroptilini (Hydroptilidae). Rev. Hydrobiol. trop., 20 (2) : 121-130.

Gibon F.-M. 1991 (1992). — Trichoptères d'Afrique Occidentale et du Cameroun. Le genre Trichosetodes (Leptoceridae). Bull. Soc. Ent. Fr., 96 (4) : 347-353.

Gibon F.-M. 1992. - Trichoptères d'Afrique Occidentale et du Cameroun. Les Ecnomus du groupe natalensis (Ecnomidae). Bull. Mus. natl. Hist. nat., section A, 14 (3-4) : 705-743.

Gibon F.-M. Sous presse. - Trichoptera from West Africa and Cameroon. The genus Chimarra.

Gibon F.-M. \& Statzner B. 1985. - Longitudinal zonation of lotic insects in the Bandama River System (Ivory Coast). Hydrobiologia, $122: 61-64$.

Guenda W. 1985. - Hydrobiologie d'un cours d'eau temporaire en zone soudanienne : la Volta Rouge (Burkina Faso Ghana). Relation avec les traitements chimiques antisimuljdjens. Thèse de 3 e cycle, Université d'Aix-Marseille : $193 \mathrm{p}$.

Guenda W. 1986-1987. - Evaluation de la toxicité de l'Abate sur les macroinvertébrés de la Volta Rouge (Burkina Faso Ghana), dans le cadre du contrôle de l'onchocercose en Afrique occidentale. Bull. I.F.A.N., ser. A, 46 (3-4) : 333-349.

Guenda $W$. sous presse. - Contribution à l'étude des Trichoptères de la Volta rouge (Afrique occidentale). I : notes sur les Hydropsychinae. Bull. I.F.A.N., ser. A, 47.

Kelley R.W. 1984. - Phylogeny, morphology and classification of the micro-caddisfly genus Oxyethira Eaton. Trans. Am. ent. Soc., 111 : 223-253.

Kimmins D.E. 1957. - Notes on the Psychomyidae (Trichoptera) from the african mainland (south of the Mediterranean region) with particular reference to the genera Ecnomus and Psychomyiellodes. Trans. R. ent. Soc. Lond., 109 (8): 249-273.
Le Berre R. 1966. - Contribution à l'étude biologique de Simulium damnosum Théobal, 1903 (Diptera : Simuliidae). Mém ORSTOM, $\mathrm{n}^{\circ} 17: 204 \mathrm{p}$.

Marlier G. 1978. - Sur une collection de Trichoptères d'Afrique occidentale. Rev. Zool. Bot. afr., $92: 238-302$.

Marshall J.E. 1979. - A review of the genera of the Hydroptilidae (Trichoptera). Bull. Br. Mus. nat. Hist. (Ent.), 39 (3) : 135-239.

Quillévéré D., Pendriez B. 1975. - Etude du complexe Simuiium damnosum en Afrique de l'Ouest II - Répartition géographique des cytotypes en Côte d'Ivoire. Cah. ORSTOM, ser. Ent. med. et Parasitol., 13 (3) : 165-172.

Quillévéré D. 1979. - Contribution à l'étude des caractéristiques taxonomiques, bioécologiques et vectrices des membres du complexe Simulium damnosum présents en Côte d'Ivoire. Trav. et Doc. ORSTOM, n 109, 304 p.

Statzmer B. 1984. - Keys to adult and immature Hydropsychinae in the Ivory coast (West Africa) with notes on their taxonomy and distribution. Spixiana, 7 (1) : 23-50.

Statzner B. \& Gibon F.-M. 1984. - Keys to adult and immature Macronematine (Insecta, Trichoptera) from the Ivory coast (West Africa) with notes on their taxonomy and distribution. Rev. Hydrobiol, trop., 17 (2) : 129-151.

Wasson J.-G. 1989. - Eléments pour une typologie fonctionnelle des eaux courantes : 1 . Revue critique de quelques approches existantes. Bull. Ecol., 20 (2) : 109-127.

Yameogo L., Lévèque C., Traore K. \& Fairhurst C.P. 1988. Dix ans de surveillance de la faune aquatique des rivières d'Afrique de l'Ouest traitées contre les Simulies (Diptera, Simuliidae), agents vecteurs de l'Onchocercose humaine. Naturaliste Can. (Rev. Ecol. Syst.), 115 : 287-298.

Zwick P. 1992. - Stream habitat fragmentation - a threat to biodiversity. Biodiversity and Conservation, 1 : 80-97.

Planche I.

Fig. 1 à 5. Cheumatopsyche tournii n. sp. : 1. vue latérale des genitalia, 2. vue dorsale du dixième segment abdominal (spécimen du Burkina), 3. appendice inférieur, 4. vue dorsale du dixième segment abdominal (spécimen du Cameroun), 5. vue latérale du dixième segment abdominal (spécimen du Cameroun).

Fig. 6 à 9. Cheumatopsyche digitata (Mosely), $1935: 6$. vue latérale du dixième segment abdominal (spécimen du Baoulé), 7. vue dorsale du dixième segment abdominal (spécimen du Niandan), 8, vue latérale du dixième segment abdominal (spécimen du Niandan), 9. appendice inférieur.

Fig. 10. Cheumatopsyche macentae n. sp., appendice inférieur.

Fig. 11. Cheumatopsyche afra (Mosely), 1935, apprendice inférieur, échelle : 0,1 mm.

Plate I.

Fig. 1 to 5. Cheumatopsyche tournii n. sp.: 1. latéral view of the genitalia, 2. dorsal view of the tenth abdominal segment (specimen from Burkina), 3. inferior appendage, 4. dorsal view of the tenth abdominal segment (specimen from Cameroon), 5 . lateral view of the tenth abdominal segment (specimen from Cameroon).

Fig. 6 to 9. Cheumatopsyche digitata (Mosely), 1935:6. lateral view of the tenth abdominal segment (specimen from the Baoulé), 7. dorsal view of the tenth abdominal segment (specimen from the Niandan), 8. latéral view of the tenth abdominal segment (specimen from the Niandam), 9. inferior appendage.

Fig. 10. Cheumatopsyche macentae $\mathrm{n}$. sp., inferior appendage.

Fig. 11. Cheumatopsyche afra (Mosely), 1935, inferior appendage ; scale : 0,1 mm. 


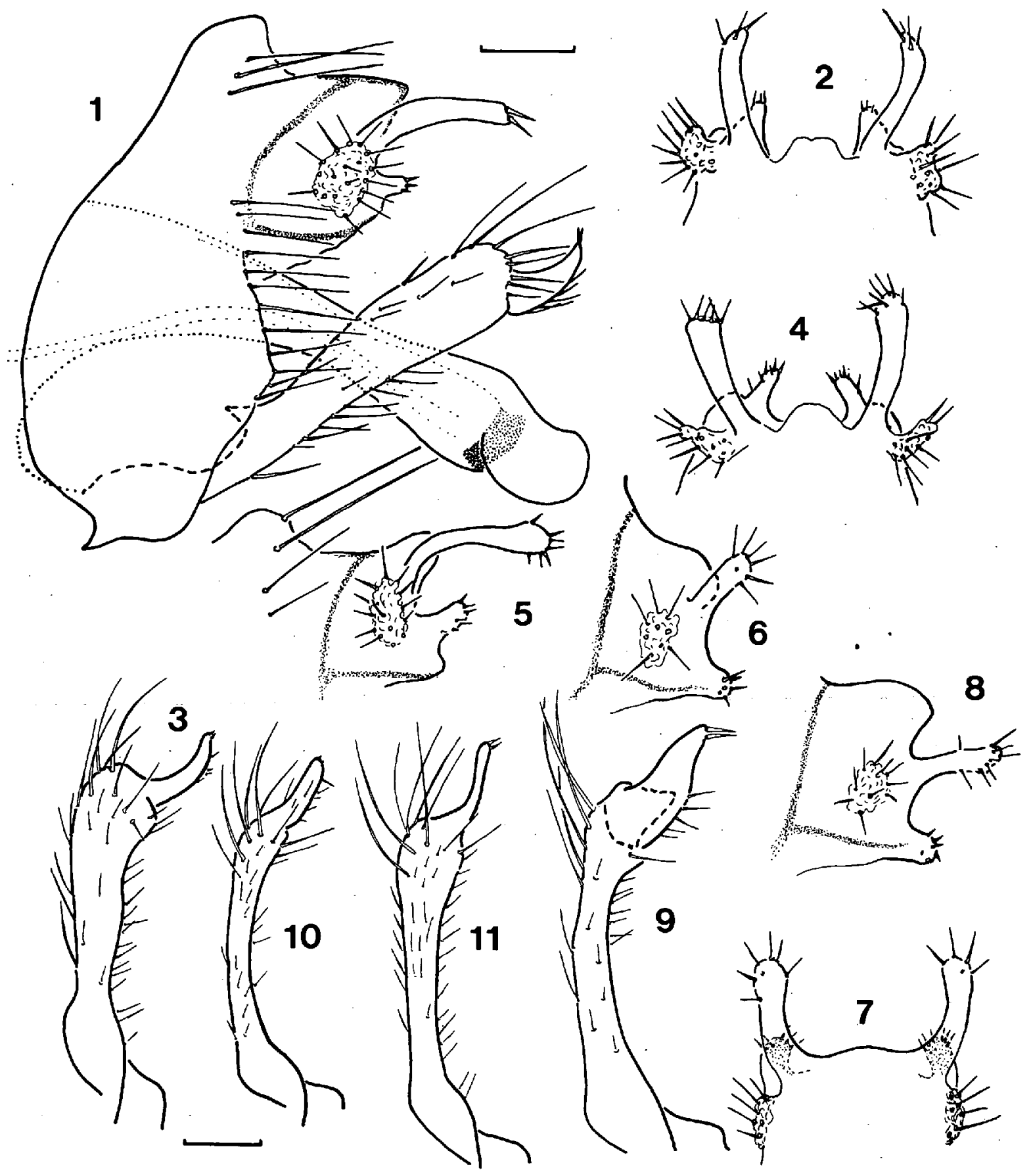

Planche I.

Plate I. 


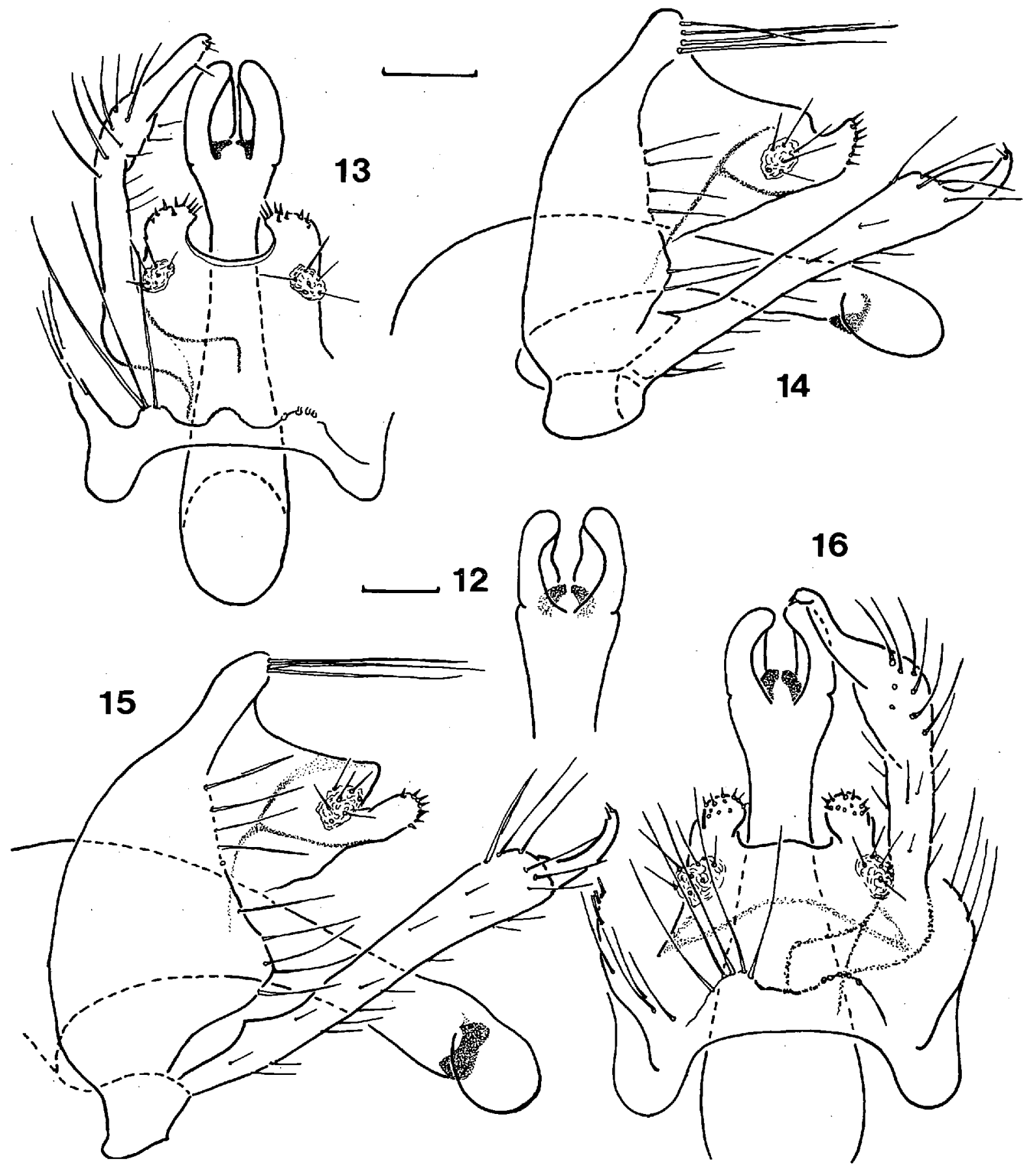

Planche II.

Fig. 12. Cheumatopsyche tournii: vue dorsale de l'apex de l'appareil phallique ; Fig. 13 et 14. Cheumatopsyche macentae : 13. vue dorsale partielle des genitalia, 14. vue latérale des genitalia; Fig. 15 et 16. Cheumatopsyche afra : 15. vue latérale des genitalia, 16. vue dorsale partielle des genitalia ; échelle : $0,1 \mathrm{~mm}$.

Plate II.

Fig. 12. Cheumatopsyche tournii : apex of the phallic apparatus (dorsal view) ; Fig. 13 and 14. Cheumatopsyche macentae : 13. dorsal view of the genitalia (part), 14. lateral view of the genitalia ; Fig. 15 and 16. Cheumatopsyche afra : 15. lateral view of the genitalia, 16. dorsal view of the genitalia (part); scale : $0,1 \mathrm{~mm}$. 


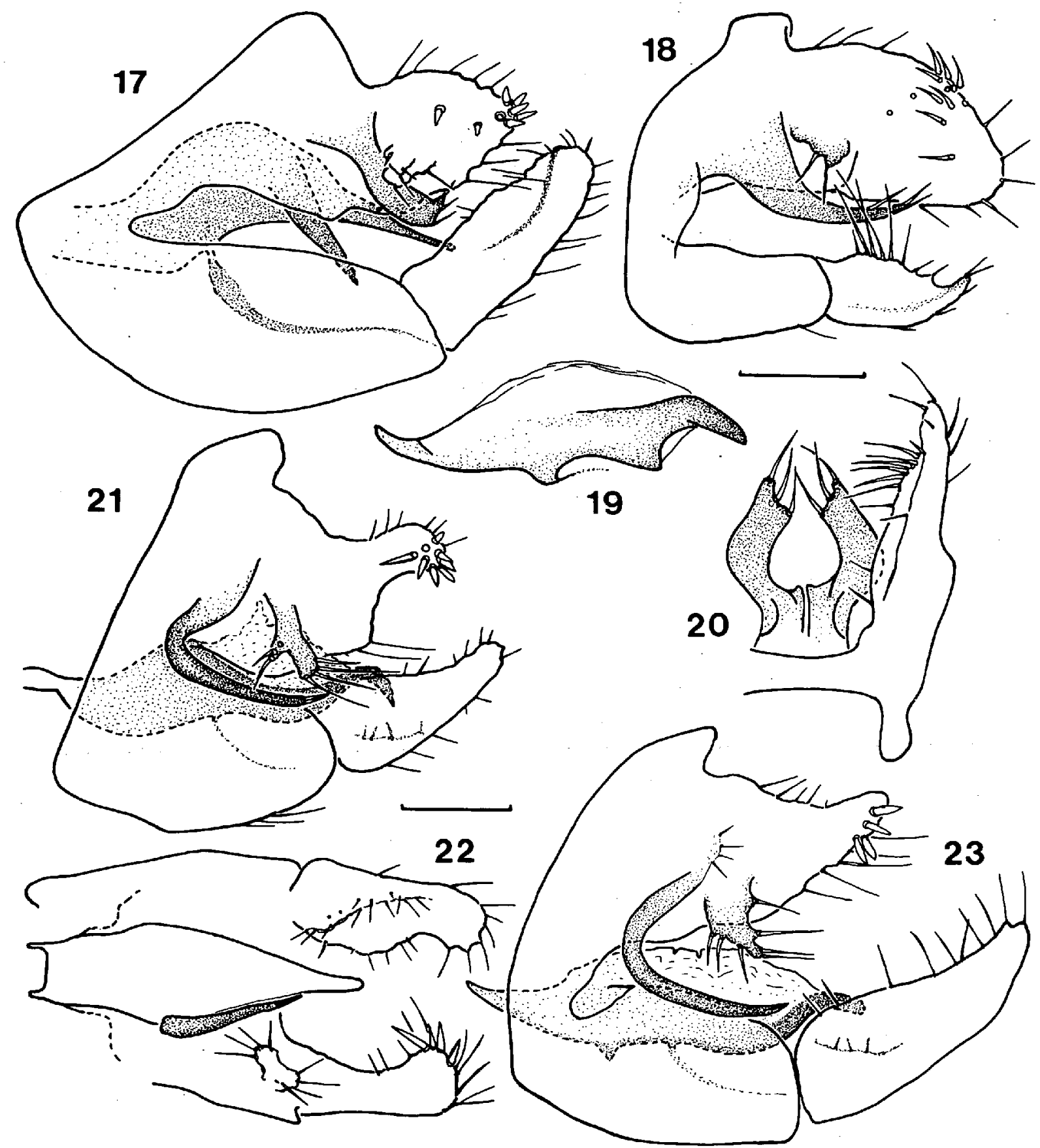

Planche III.

Fig. 17. Ecnomus ulmeri Mosely, 1932 : vue latérale des genitalia ; Fig. 18 à 20. Ecnomus wendmanegrei n. sp. : 18. vue latérale des genitalia (appareil phallique ôté), 19. vue latérale de l'appareil phallique, 20. vue dorsale partielle du dixième segment abdominal ; Fig. 21 et 22. Ecnomus dinderessoi n. sp. : 21, vue latéralè des genitalia, 22. vue dórsale partielle des genitalia ; Fig. 23. Ecnomus dispar Kimmins, 1957 (Cameroun): vue latérale des genitalia ; échelle : 0,1 mm.

Plate III.

Fig. 17. Ecnomus ulmeri Mosely, 1932 : lateral view of the genitalia ; Fig. 18 to 20. Ecnomus wendmanegrei n. sp., 18. lateral view of the genitalia (phallic apparatus removed), 19. lateral view of the phallic apparatus, 20. dorsal view of the tenth abdominal segment (part) ; Fig. 21 and 22. Ecnomus dinderessoi n. sp., 21. lateral view of the genitalia, 22. dorsal view of the genitalia (part) ; Fig. 23. Ecnomus dispar Kimmins, 1957 (Cameroon), lateral view of the genitalia ; scale : 0,1 mm. 

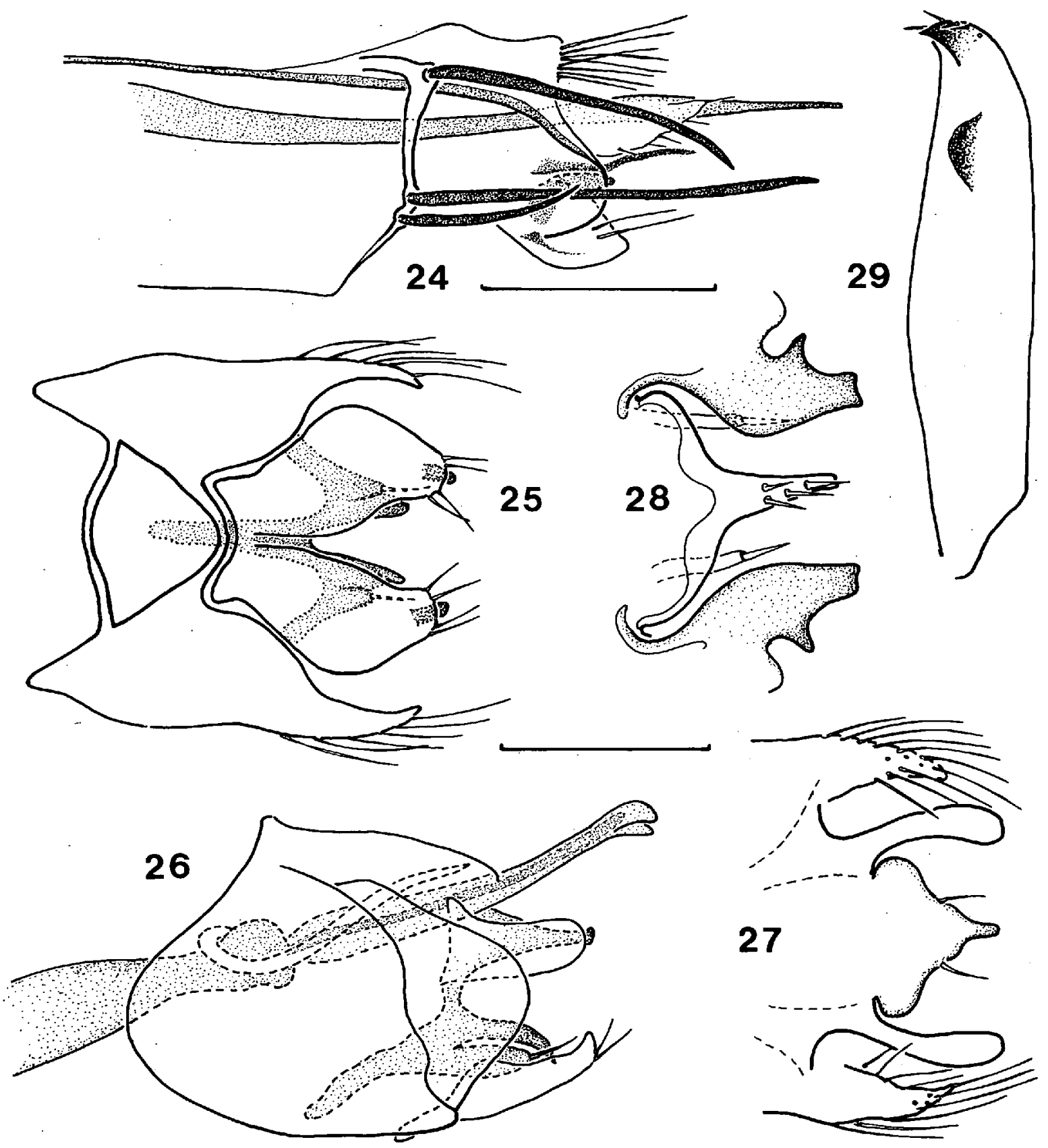

Planche IV.

Fig. 24. Catoxyethira graboensis Gibon, 1985 (spécimen de la Comoé) : vue latérale partielle des genitalia ; Fig. 25 à 27. Ugandatrichia lerabae n. sp. : 25 . vue ventrale partielle des genitalia, 26 . vue latérale des genitalia, 27 . vue dorsale de la plaque sous-génitale ; Fig. 28 et 19. Ugandatrichia atakpamensis Gibon, 1987 (spécimen de la Comoé), 28. vue dorsale de la plaque sous-génitale, 29. vue dorsale d'un appendice inférieur ; échelle : $0,1 \mathrm{~mm}$.

Plate IV.

Fig. 24. Catoxyethira graboensis Gibon, 1985 (specimen from the Comoé), lateral view of the genitalia (part) ; Fig. 25 to 27. Ugandatrichia lerabae n. sp., 25. ventral view of the genitalia (part), 26. lateral view of the genitalia, 27. dorsal view of the subgenital plate ; Fig. 28 and 29. Ugandatrichia atakpamensis Gibon, 1987 (specimen from the Comoé), 28. dorsal view of the subgenital plate, 29. dorsal view of an inferior appendage; scale : $0,1 \mathrm{~mm}$. 

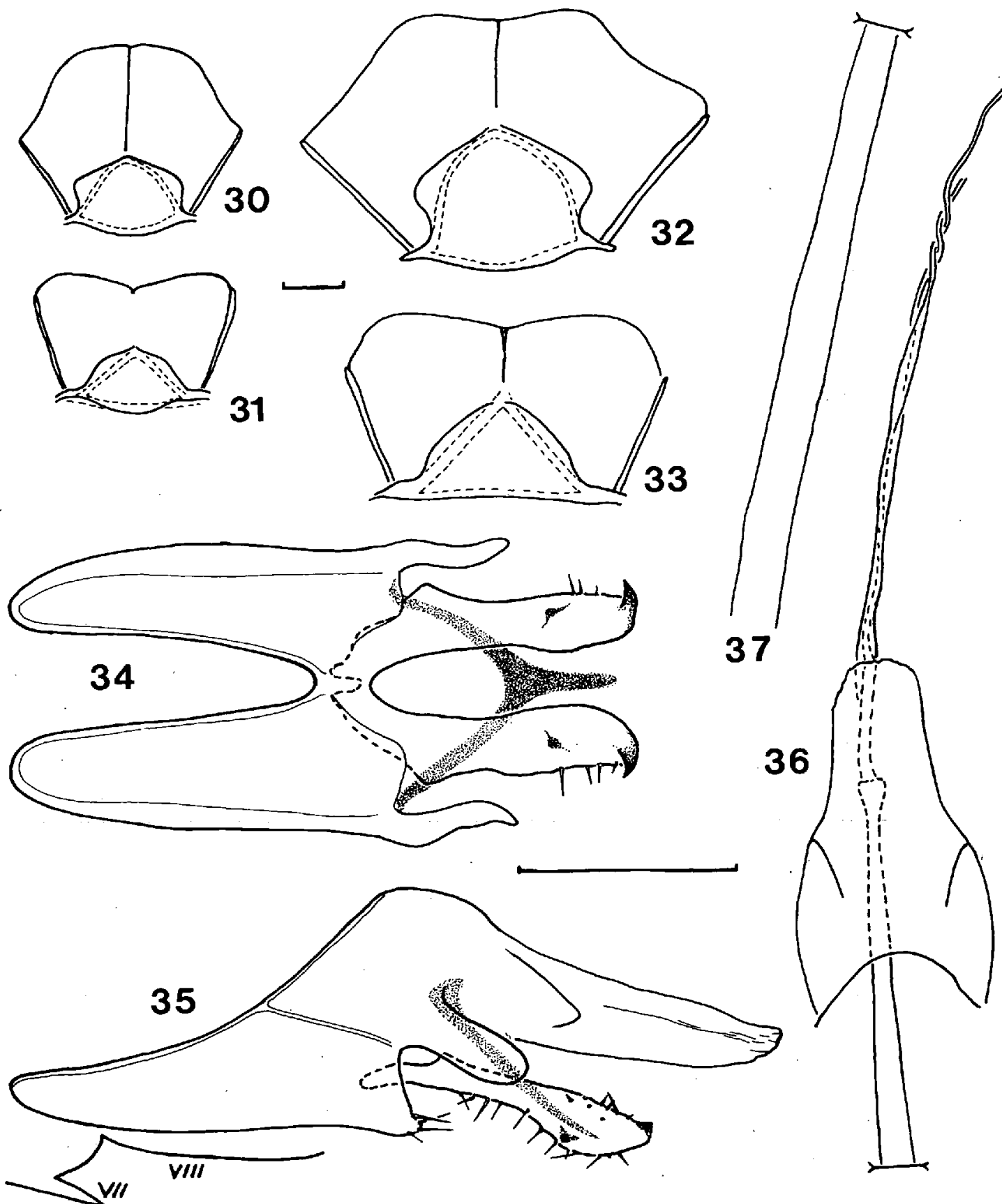

Planche V.

Fig. 30 et 31. Ugandatrichia lerabae : 30. vue dorsale du deuxième segment thoracique, 31 . vue dorsale du troisième segment thoracique ; Fig. 32 et 33. Ugandatrichia yameogoi Gibon, $1987: 32$. vue dorsale du deuxième segment thoracique, 33. vue dorsale du troisième segment thoracique ; Fig. 34 à 37. Hydroptila maoae n. sp. : 34. vue ventrale partielle des genitalia, 35. vue latérale des genitalia, 36. vue dorsale de l'appareil phallique et du dixième segment abdominal, 37. base de l'appareil phallique ; échelle : $0,1 \mathrm{~mm}$.

Plate V.

Fig. 30 and 31. Ugandatrichia lerabae : 30. dorsal view of the second thoracic segment, 31 . dorsal view of the third thoracic segment ; Fig. 32 and 33. Ugandatrichia yameogoi Gibon, $1987: 32$. dorsal view of the second thoracic segment, 33. dorsal view of the third thoracic segment ; Fig. 34 to 37. Hydroptila maoae n. sp. : 34. ventral view of the genitalia (part), 35. lateral view of the genitalia, 36. dorsal view of the phallic apparatus and tenth abdominal segment, 37 . base of the phallic apparatus ; scale : $0,1 \mathrm{~mm}$. 


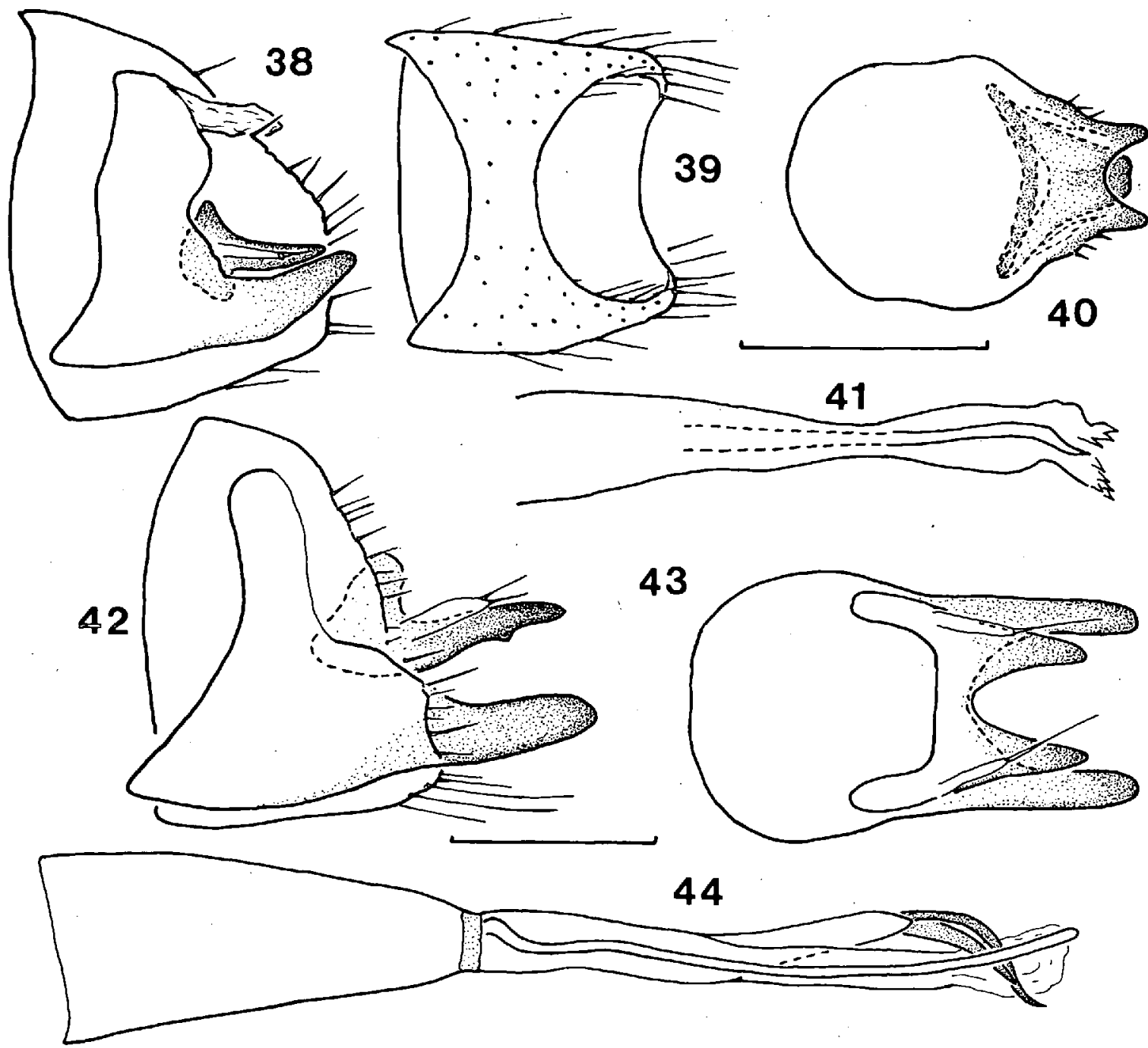

Planche. VI.

Fig. 38 à 41 . Oxyethira burkina n. sp. : 38. vue latérale des genitalia, 39. vue dorsale du huitième segment abdominal, 40. vue ventrale du neuvième segment et de la plaque sous-génitale, 41. vue dorsale de l'appareil phallique; Fig. 42 à 44 . Oxyethira tenei n. sp. : 42. vue latérale des genitalia, 43. vue dorsale du neuvième segment et de la plaque sous-génitale, 44 . vue dorsale de l'appareil phallique ; échelle : $0,1 \mathrm{~mm}$.

Plate VI.

Fig. 38 to 41 . Oxyethira burkina n. sp. : 38. lateral view of the genitalia, 39. dorsal view of the eighth abdominal segment, 40 . ventral view of the ninth segment and subgenital plate, 41 . dorsal view of the phallic apparatus; Fig. 42 to 44 . Oxyethira tenei $\mathrm{n}$. sp. : 42. lateral view of the genitalia, 43. dorsal view of the ninth segment and subgenital plate, 44 . dorsal view of the phallic apparatus; scale : $0,1 \mathrm{~mm}$. 


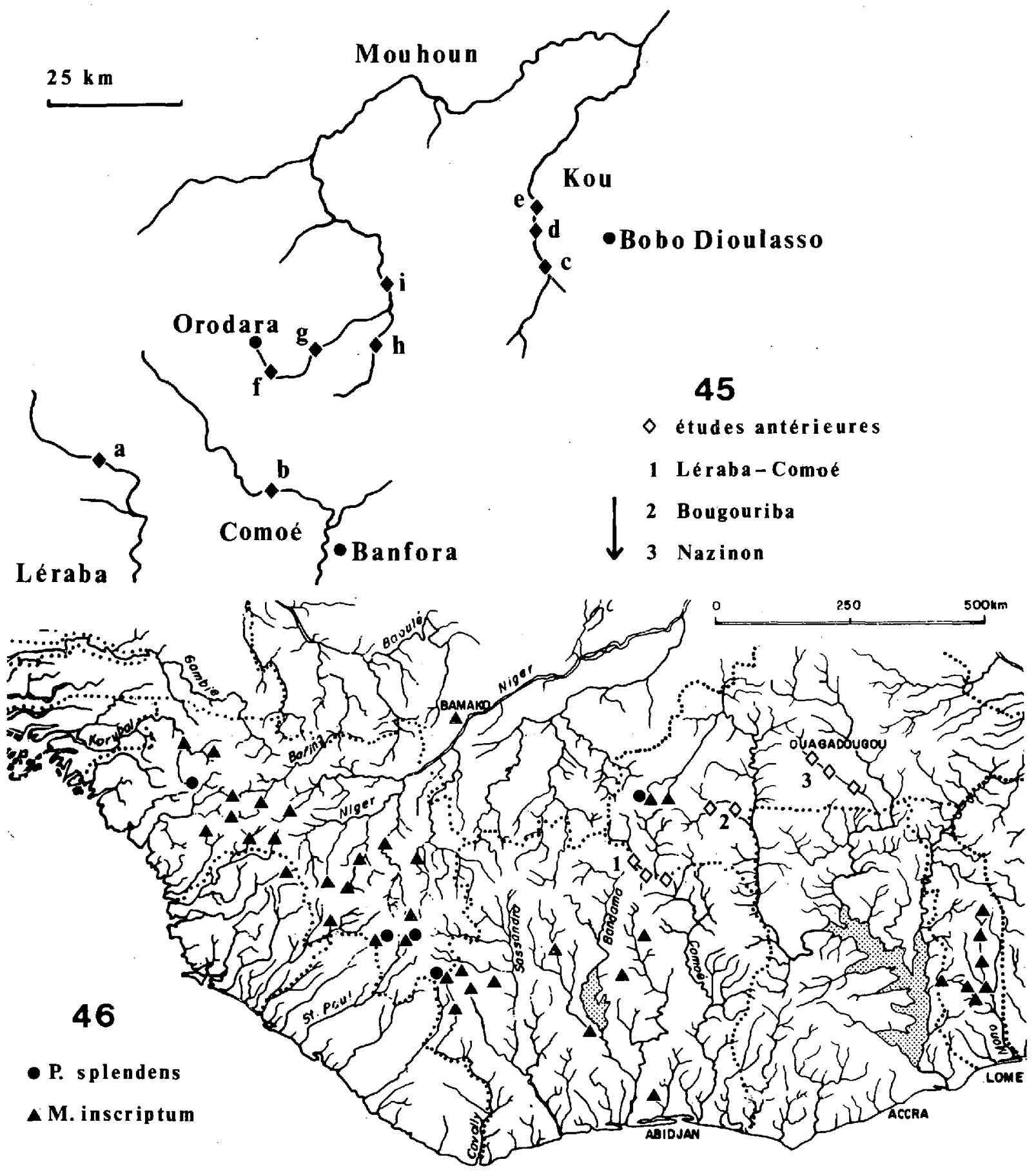

Fig. 45. Localisations des sites étudiés dans le sud-ouest du Burkina Faso.

Fig. 46. Localisation des captures de Protomacronema splendens et de Macrostemum inscriptum en Afrique occidentale.

Fig. 45. Location of the study sites in the South-Western part of Burkina Faso.

Fig. 46. Captures of Protomacronema splendens and of Macrostemum inscriptum in Western Africa: 

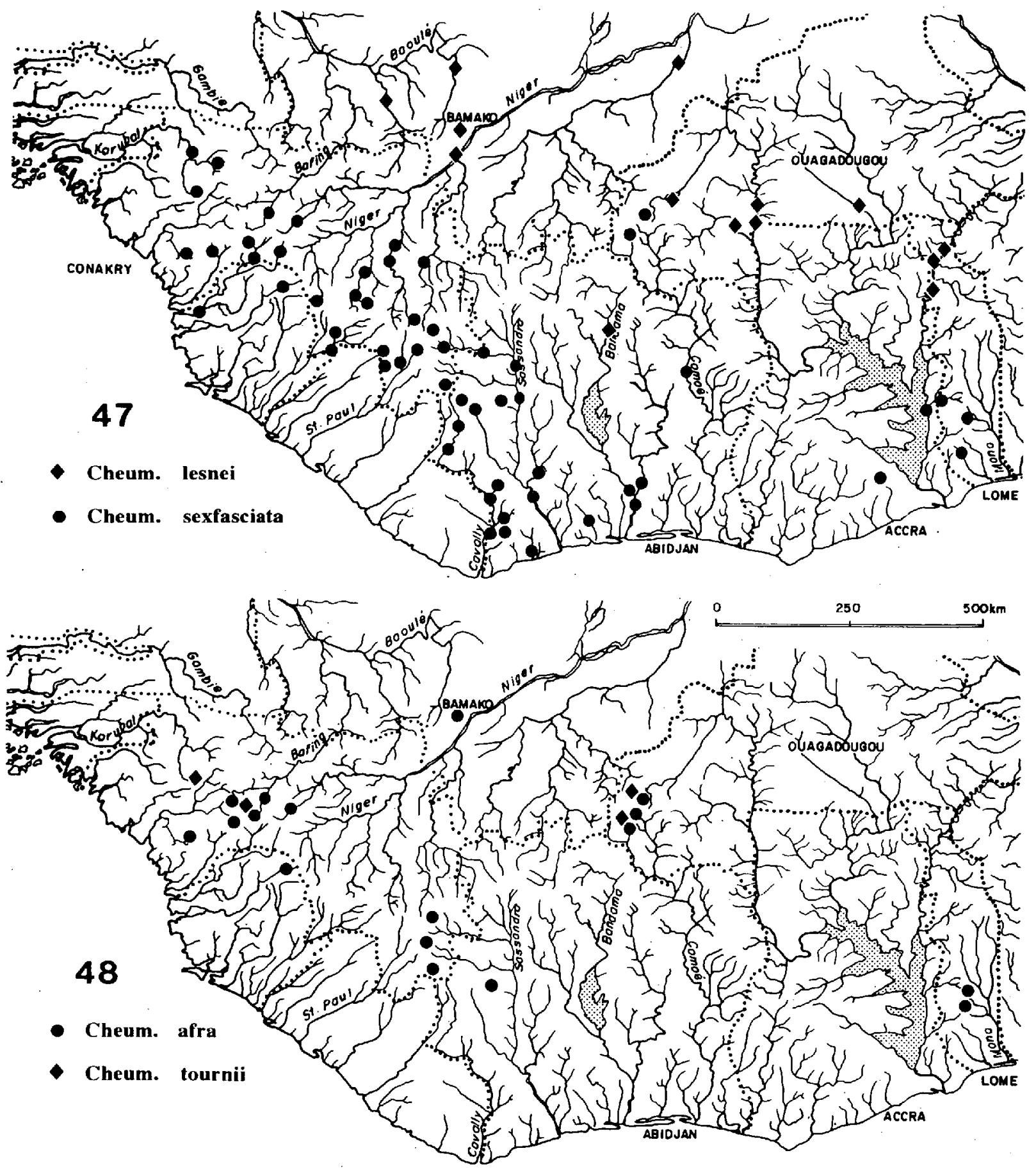

Fig. 47. Localisation en Afrique occidentale des captures de Cheumatopsyche lesnei (Mosely), 1932 et de Cheumatopsyche sexfasciata (Ulmer), 1904.

Fig. 48. Localisation en Afrique occidentale des captures de Cheumatopsyche afra et de Cheumatopsyche tournii.

Fig. 47. Captures of Cheumatopsyche lesnei (Mosely), 1932 and of Cheumatopsyche sexfasciata (Ulmer), 1994 in Western Africa.

Fig. 48. Captures of Cheumatopsyche afra and of Cheumatopsyche tournii in Western Africa. 

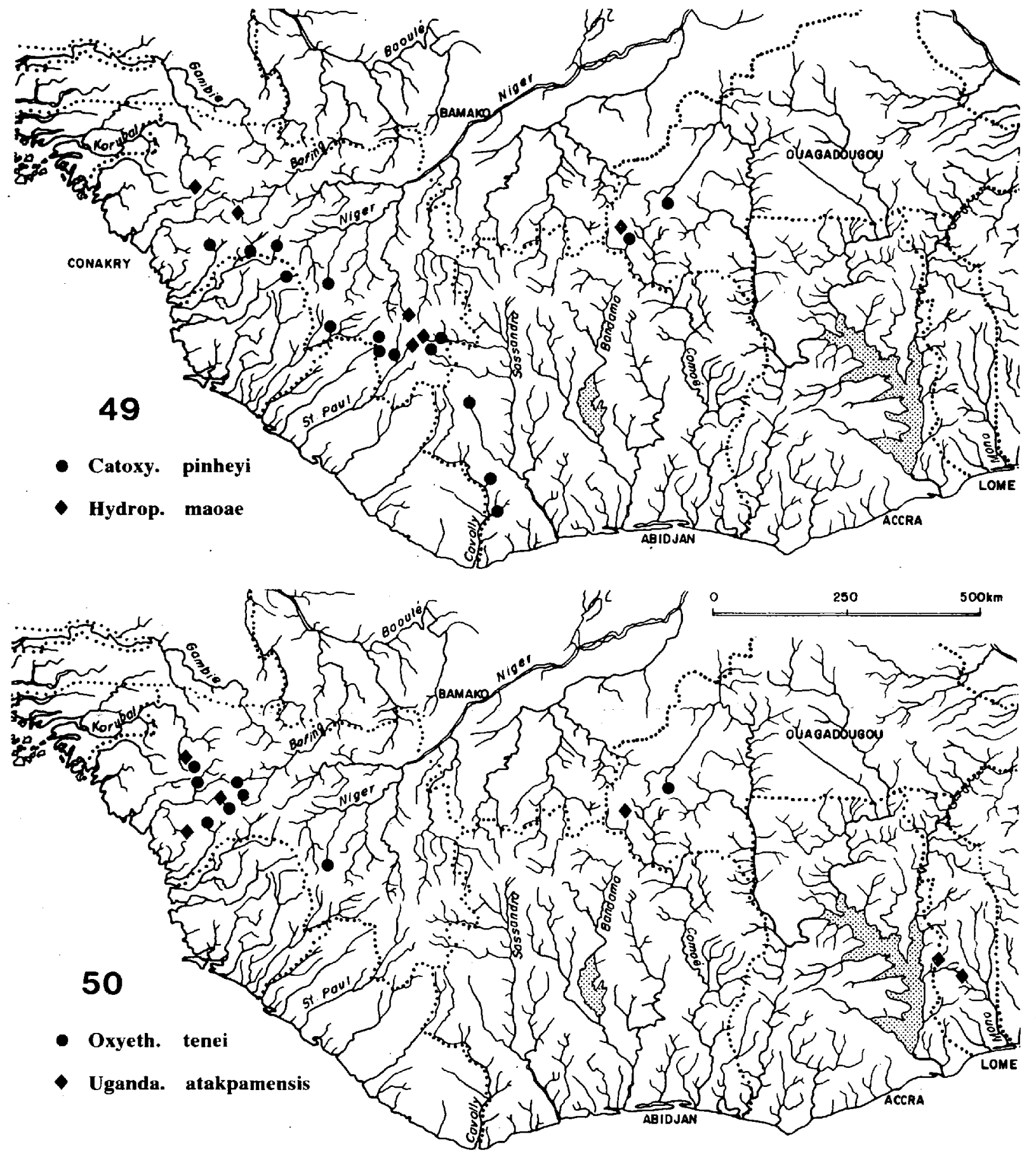

Fig. 49. Localisation en Afrique occidentale des captures de Catoxyethira pinheyi et d'Hydroptila maoae. Fig. 50. Localisation en Afrique occidentale des captures d'Oxyethira tenei et d'Ugandatrichia atakpamensis.

Fig. 49. Captures of Catoxyethira pinheyi and of Hydroptila maoae in Western Africa.

Fig. 50. Captures of Oxyethira tenei and Ugandatrichia atakpamensis in Werten Africa. 